\title{
UTILIZAÇÃO DE TECNOLOGIAS EDUCACIONAIS DIGITAIS NO ENSINO DE ENFERMAGEM
}

\author{
THE USE OF DIGITAL EDUCATIONAL TECHNOLOGIES \\ IN NURSING EDUCATION
}

\section{UTILIZACIÓN DE TECNOLOGÍAS EDUCACIONALES DIGITALES EN LA ENSEÑANZA DE ENFERMERÍA}

\author{
Ana Luísa Petersen Cogo* \\ Eva Néri Rubim Pedro** \\ Ana Paula Scheffer Schell da Silva ${ }^{* * *}$ \\ Elcilene Andreíne Terra Durgante Alves ${ }^{* * * *}$ \\ Gabriela PetróValli ${ }^{* * * *}$
}

\begin{abstract}
RESUMO
Estudo exploratório quantitativo que teve como objetivo descrever a utilização de tecnologias educacionais digitais (TED) no ensino de enfermagem em Instituições de Ensino Superior (IES). Amostra constituída por dez coordenadores e 55 professores de cursos de Enfermagem do Estado do Rio Grande do Sul. Os dados foram analisados pela estatística descritiva. Os resultados apontaram que houve apoio para desenvolvimento de TED em 90\% das Instituições, das quais 77,8\% têm setores especializados na produção de material. Três (30\%) cursos de graduação oferecem disciplina a distância e 47,3\% dos professores fizeram curso de qualificação. O recurso mais usado pelos professores em aula é o vídeo (82,4\%). Concluiu-se que as IES oferecem infraestrutura tecnológica e/ou pedagógica para o atendimento da demanda crescente no uso da informática no ensino, no entanto, ações em educação a distância ainda são limitadas no ensino da Enfermagem, destacando o uso das TED no ensino presencial.
\end{abstract}

Palavras chave: Educação em Enfermagem,tecnologia educacional, educação a distância, internet.

\footnotetext{
ABSTRACT

Quantitative exploratory study aimed to describe the use of digital educational technologies (DET) on nursing education in Under Graduation Institutions (UGI). Sample constituted of ten coordinators and 55 teachers of Nursing Courses in the State of Rio Grande do Sul. Data were analyzed using descriptive statistics. The results showed that there was support for development of DET in $90 \%$ of the Institutions, of which $77.8 \%$ have special-

* Enfermeira, Professora do Departamento de Enfermagem Médico-Cirúrgica em Enfermagem da Universidade Federal do Rio Grande do Sul, Brasil. Email: analuisa@enf.ufrgs.br

** Enfermeira, Professorado Departamento de Enfermagem Materno-Infantil em Enfermagem da Universidade Federal do Rio Grande do Sul, Brasil. Email: evapedro@enf.ufrgs.br

${ }^{* * *}$ Enfermeira, Professora do Departamento de Enfermagem da Universidade Federal de Ciências da Saúde de Porto Alegre, Brasil. Email: anaschell@gmail.com

${ }_{* * * *}$ Enfermeira, Mestranda do Programa de Pós-Graduação em Enfermagem da Universidade Federal do Rio Grande do Sul, Brasil. Email: elcilene.alves@hps.prefpoa.com.br

${ }^{* * * * *}$ Enfermeira. Mestranda do Programa de Pós-Graduação em Enfermagem da Universidade Federal do Rio Grande do Sul. Email: gabi130987@gmail.com
} 
ized sectors in the production of material. Three (30\%) under graduation programs offer distance courses and $47.3 \%$ of the teachers have qualification course. The feature most used by teachers in class is the video (82.4\%). It was concluded that UGI offer technological infrastructure and/or pedagogic to meet the growing demand in the use of computers in teaching, however, actions in education at distance are still limited in the teaching of nursing, highlighting the use of DET in classroom teaching.

Key words: Education, Nursing, educational technology, education, distance, internet.

\section{RESUMEN}

Estudio exploratorio cuantitativo cuyo objetivo fue describir la utilización de tecnologías educacionales digitales (TED) en la enseñanza de Enfermería en Instituciones de Educación Superior (IES). Muestra constituida por diez coordinadores y 55 profesores de cursos de Enfermería del Estado de Rio Grande do Sul. Los datos fueron analizados utilizando estadística descriptiva. Los resultados muestran que hubo apoyo para desarrollo de TED en $90 \%$ de las Instituciones, de las cuales $77,8 \%$ tienen sectores especializados en la producción de material. Tres (30\%) cursos de graduación ofrecen asignaturas a distancia y 47,3\% de los profesores hicieron curso de cualificación. El recurso más usado por los profesores en clase es el vídeo (82,4\%). Se concluyó que las IES ofrecen infraestructura tecnológica y/o pedagógica para atender la demanda creciente en el uso de la informática en la enseñanza, sin embargo, acciones en educación a distancia aún son limitadas en la enseñanza de la Enfermería, destacando el uso de las TED en la enseñanza presencial.

Palabras clave: Educación en Enfermería, tecnología educacional, educación a distancia, internet.

Fecha recepción: 29/06/12 Fecha aceptación: 23/10/13

\section{INTRODUÇÃO}

As tecnologias educacionais digitais (TED), entendidas como recursos informatizados utilizados no apoio ao ensino, tais como geradores de apresentação, vídeos, ambientes virtuais, estão sendo amplamente utilizadas pelas Instituições de Ensino Superior brasileiras nas últimas décadas. Tal fato pode ser observado em relatos de atividades de ensino mediadas por computador, tanto com estudantes de enfermagem de diferentes níveis acadêmicos, como na educação permanente com enfermeiros $(1,2)$.

Assim, na primeira década do século XXI observa-se, não somente uma expansão das iniciativas em educação a distância (EAD) na área da enfermagem promovidas pelas instituições de ensino superior para diferentes públicos, mas também a utilização da informática nas disciplinas presenciais. Esses fatos são evidenciados pelo desenvolvimento de cursos online mediados por ambientes virtuais de aprendizagem, pela criação de disciplinas na modalidade a distância e de objetos educacionais digitais em diferentes contextos do ensino de enfermagem (3-6).

Essas iniciativas são consideradas importantes para dinamizarem o ensino, desenvolverem projetos ativos de aprendizagem e competências dos futuros enfermeiros para a utilização das tecnologias da informação e da comunicação preconizadas pelas diretrizes curriculares nacionais brasileiras (7, 8). O emprego de tais recursos tecnológicos possui uma dupla função, a de flexibilizar o ensino de enfermagem por meio de uma proposta pedagógica ativa e a de desenvolver competências com os estudantes no uso de diferentes recursos de informática.

As competências, na perspectiva construtivista, são entendidas como conhecimentos, habilidades e atitudes mobilizados para a resolução de problemas em determinada situação e contexto, sendo esta sua afinidade com 
as TED e os métodos ativos de aprendizagem (9).

Apesar dos dados (3-7) indicarem uma expansão da informática no ensino, estudos sinalizam a baixa oferta de cursos lato sensu (10) e de graduação em Enfermagem (11) na modalidade a distância no Brasil. Isso demonstra que as instituições de ensino de enfermagem estão desenvolvendo ações específicas, existindo poucas opções de cursos de formação complementar aos enfermeiros.

Da mesma forma, a produção científica na Enfermagem tem seu foco direcionado para o estudo curricular, das práticas docentes, do processo de ensino-aprendizagem, das vivências dos estudantes e a avaliação do ensino (7), não tendo sido explorada a organização das Instituições de ensino superior para utilizarem as TED.

Frente ao exposto, questionam-se quais tem sido as ações nos cursos de ensino superior de enfermagem no Estado do Rio Grande do Sul, Brasil, para o desenvolvimento e a aplicação das TED? O conhecimento das atividades e a forma como as instituições estão organizando-se para implantar ações utilizando TED no ensino de enfermagem nas modalidades presenciais e a distância, irão contribuir para que possam ser desenvolvidas estratégias interinstitucionais e interdisciplinares para a formação e a atualização dos professores de enfermagem em nível estadual.

Assim, o objetivo geral da presente investigação foi o de descrever a utilização de TED no ensino de enfermagem em Instituições de Ensino Superior. Os objetivos específicos foram o de identificar as propostas institucionais de utilização de TED que estão sendo desenvolvidas junto aos cursos de graduação e de pós-graduação em enfermagem no Estado do Rio Grande do Sul e também, identificar os recursos de informática com os seus contextos pedagógicos utilizados por professores de enfermagem nessas instituições nas modalidades presenciais e a distância.

\section{MATERIAL E MÉTODO}

Trata-se de uma pesquisa exploratória empregando uma abordagem quantitativa (12) referente às aplicações tecnológicas no ensino de enfermagem. No Estado do Rio Grande do Sul, segundo os registros Ministério da Educação (MEC) em 2009, encontravam-se em funcionamento 29 faculdades ou escolas de Enfermagem. Procurou-se contatar todas as instituições. No entanto, ocorreram dificuldades em ter acesso a diretores ou coordenadores de alguns cursos por ausência ou imprecisão de informações nos sites das instituições.

Assim, aceitaram a participar do estudo, constituindo uma amostra por conveniência, dez diretores ou coordenadores das faculdades ou escolas de Enfermagem e 55 professores de disciplinas profissionalizantes de enfermagem destas instituições que utilizavam TED nas modalidades presenciais ou a distância. Foram excluídos da amostra os professores desses cursos que não eram graduados em enfermagem ou que ministravam aulas em áreas afins, como exemplo, anatomia.

Considerou-se como TED, no presente estudo, a utilização de ambiente virtual de aprendizagem, de videoconferência, de fórum de discussão, de lista de discussão por correio eletrônico, de bate-papo, de redes sociais digitais e objetos educacionais digitais.

A coleta de dados ocorreu entre novembro de 2010 e junho de 2011. Os participantes foram convidados por meio de correio eletrônico, contato telefônico ou pessoalmente por material impresso. Os dados foram organizados em planilha eletrônica e a análise ocorreu pela estatística descritiva, com as variáveis categóricas sendo descritas por frequência absoluta e relativa.

Foram observados os aspecto éticos em pesquisa com seres humanos, iniciando-se a coleta de dados após as tramitações junto 
à Comissão de Pesquisa da Unidade Acadêmica e ao Comitê de Ética em Pesquisa da Universidade (aprovação de número 18455). A devolução do questionário, digitalizado ou impresso, foi considerado o aceite em participar do estudo. Aos participantes foi assegurado o anonimato e a possibilidade de retirarem-se do estudo a qualquer momento. As informações serão armazenadas por cinco anos com posterior descarte (13).

\section{RESULTADOS}

Os dados serão apresentados em dois momentos, o primeiro refere-se às propostas institucionais na utilização de TED na Enfermagem e o segundo tratará da utilização de TED por professores no ensino superior de Enfermagem.

As propostas institucionais na utilização de TED nos Cursos de Enfermagem foram relatadas por dez (100\%) coordenadores ou diretores de ensino de Cursos de Enfermagem, sendo quatro (40\%) de instituições de Porto Alegre e da região metropolitana, seis (60\%) do interior do Estado do Rio Grande do Sul. As Instituições oferecem o Curso de Graduação em Enfermagem, sendo que além deste, oito $(80 \%)$ realizam Cursos de lato sensu, duas (20\%) Cursos stricto sensu e seis (60\%) Cursos de extensão e/ou de aperfeiçoamento.

O desenvolvimento de ações utilizando TED recebe o apoio em nove (90\%) das instituições de ensino dos participantes do estudo. Entre os recursos oferecidos por estas estão a orientação e o planejamento de atividades mediadas pelo computador $(77,8 \%)$, existência de setor especializado para a realização de atividades usando tecnologias educacionais digitais $(77,8 \%)$, disponibilização de recursos para desenvolver as ações $(66,7 \%)$, cursos de capacitação para uso das tecnologias $(55,6 \%)$ e presença de setor especializado para desenvolver objetos educa- cionais $(55,6 \%)$.

A oferta de disciplinas na modalidade EAD ocorre em três (30\%) Cursos de Graduação em Enfermagem pesquisados e em um (10\%) Curso de Pós-Graduação lato sensu. No entanto, os coordenadores dessas nove (90\%) Instituições referiram que são utilizadas TED em disciplinas presenciais. No caso de cursos de extensão e/ou aperfeiçoamento na modalidade $\mathrm{EAD}$, esses são realizadas em uma (10\%) das instituições estudas.

Os coordenadores dos cursos destacaram como propostas que poderiam ser implementadas em suas instituições, o investimento no desenvolvimento tecnológico (40\%). Neste aspecto, incluíram a criação de laboratórios para uso dos alunos, o desenvolvimento de objetos de aprendizagem, a aquisição de equipamentos e de software, a contratação de bolsistas, o apoio técnico e financeiro para os projetos de desenvolvimento.

Também destacaram a importância da capacitação dos docentes e dos discentes no uso das tecnologias disponíveis (30\%), a formação pedagógica do docente (20\%), a criação de uma rede de Instituições de Ensino Superior em Enfermagem para desenvolvimento de projetos conjuntos e trocas de experiências nessa área (20\%), e a proposição de cursos stricto sensu na modalidade EAD com aprovação pela Coordenação de Aperfeiçoamento de Pessoal do Ensino Superior (CAPES) para atender os docentes do interior do estado (10\%).

A utilização de TED no Ensino Superior de Enfermagem, das instituições que aceitaram participar do estudo, foi relatada por 55 (100\%) professores das instituições participantes, sendo 33 (60\%) de Porto Alegre, capital do Estado. Desses professores, 27 $(49,1 \%)$ tinham o título de doutorado e 20 $(36,4 \%)$ de mestrado. Quanto a faixa etária, ocorreu uma distribuição homogênea, predominando a de 41 a 50 anos $(29,09 \%)$. O tempo de graduado predominante foi o entre os 21 e 30 anos $(32,7 \%)$ seguido dos seis aos dez anos (25,5\%). O tempo de atuação 
como professor foi até 5 anos (34,55\%), seguido de 11 à 20 anos $(25,45 \%) 6$ à 10 anos $(21,82 \%)$ (Tabela 1$)$.
Em relação a qualificação dos professores por meio de cursos sobre TED, essa foi realizada por $26(47,3 \%)$ dos entrevistados.

Tabela 1. Caracterização dos professores das instituições participantes. Porto Alegre, 2011.

\begin{tabular}{|c|c|c|}
\hline Cidade & $\mathrm{n}$ & $\%$ \\
\hline Porto Alegre & 33 & 60,00 \\
\hline Uruguaiana & 6 & 10,91 \\
\hline Gravataí & 4 & 7,27 \\
\hline Pelotas & 4 & 7,27 \\
\hline Caxias do Sul & 3 & 5,45 \\
\hline Três de Maio & 2 & 3,64 \\
\hline Frederico Westphalen & 2 & 3,64 \\
\hline São Leopoldo & 1 & 1,82 \\
\hline \multicolumn{3}{|l|}{ Título do entrevistado } \\
\hline Doutorado & 27 & 49,09 \\
\hline Mestrado & 20 & 36,36 \\
\hline Especialização & 6 & 10,91 \\
\hline Graduação & 2 & 3,64 \\
\hline Licenciatura & - & - \\
\hline \multicolumn{3}{|l|}{ Idade } \\
\hline Até 30 anos & 13 & 23,64 \\
\hline 31 até 40 anos & 11 & 20,00 \\
\hline 41 até 50 anos & 16 & 29,09 \\
\hline 51 até 60 anos & 12 & 21,82 \\
\hline Mais de 60 anos & 3 & 5,45 \\
\hline \multicolumn{3}{|l|}{ Tempo de graduado } \\
\hline Até 5 anos & 7 & 12,73 \\
\hline 6 até 10 anos & 14 & 25,45 \\
\hline 11 até 20 anos & 5 & 9,09 \\
\hline 21 até 30 anos & 18 & 32,73 \\
\hline Mais de 30 anos & 10 & 18,18 \\
\hline Não informado & 1 & 1,82 \\
\hline \multicolumn{3}{|c|}{ Tempo de atuação como professor } \\
\hline Até 5 anos & 19 & 34,55 \\
\hline 6 até 10 anos & 12 & 21,82 \\
\hline 11 até 20 anos & 14 & 25,45 \\
\hline 21 até 30 anos & 7 & 12,73 \\
\hline Mais de 30 anos & 3 & 5,45 \\
\hline Total & 55 & 100,00 \\
\hline
\end{tabular}


Da mesma forma, $23(41,8 \%)$ dos professores referiram ter tido apoio da Instituição na qual trabalham para o desenvolvimento das TED. Dentre essas ações de apoio Institucional destacaram-se a presença de um setor de apoio na orientação e no planejamento das atividades usando tecnologias (56,5\%), a realização de cursos de qualificação na área $(52,2 \%)$ e disponibilização de recursos para desenvolver os materiais necessários $(43,5 \%)$.
O desenvolvimento de ações na modalidade EAD foi realizado por 15 professores $(27,3 \%)$, sendo todas em disciplinas do Curso de Graduação em Enfermagem. Nas atividades presenciais, as tecnologias são utilizadas por $51(92,7 \%)$ dos entrevistados. Entre os recursos mais utilizados destacaram-se vídeos $(82,4 \%)$, ambiente virtual de aprendizagem $(47,1 \%)$, fórum de discussão $(27,5 \%)$, lista de discussão por correio eletrônico $(25,5 \%)$ (Tabela 2).

Tabela 2. Utilização de TED no ensino presencial. Porto Alegre, 2011.

\begin{tabular}{lrr}
\hline Uso de TED em atividade presencial & $\mathrm{n}$ & $\%$ \\
\hline Sim & 51 & 92,73 \\
Não & 4 & 7,27 \\
\hline Caso sim, cite: & & \\
\hline Vídeos & 42 & 82,35 \\
Ambiente virtual de aprendizagem & 24 & 47,06 \\
Fórum de discussão & 14 & 27,45 \\
Lista de discussão por correio eletrônico & 13 & 25,49 \\
Videoconferência & 9 & 17,65 \\
Objetos educacionais digitais & 9 & 17,65 \\
Bate-papo & 6 & 11,76 \\
Editor de texto coletivo (wiki) & 5 & 9,80 \\
Redes sociais digitais & 4 & 7,84 \\
\hline Outro(s) & 2 & 3,92 \\
\hline
\end{tabular}

Os professores, ao serem questionados sobre qual referencial pedagógico que utilizam nas suas ações mediadas por computador, referiram predominantemente que não identificam a teoria que os subsidia $(41,8 \%)$. Muitos dos participantes do estudo (29,1\%), não responderam essa questão. Os demais referiram o construtivismo (10,9\%), a teoria da problematização $(9,1 \%)$, os métodos ativos de aprendizagem (3,6\%), o referencial crítico-reflexivo $(1,8 \%)$ e a aprendizagem baseada em problemas (1,8\%).

\section{DISCUSSÃO E CONCLUSÃO}

As dez Instituições de Ensino Superior em Enfermagem participantes do estudo demonstraram que existe uma preocupação com o desenvolvimento das TED. A criação de setores especializados no apoio técnico e pedagógico, como também a oferta de cursos para capacitar os professores, caracteriza-se por ser uma estratégia condizente com as tendências para a propagação da informática 
no ensino de enfermagem $(9,10)$.

Mesmo assim, professores de algumas dessas instituições, referiram que não contavam com o apoio Institucional nessa área. As hipóteses para essa manifestação são múltiplas, entre as quais se destacam: divulgação inadequada ou inexistente dos recursos disponíveis, a falta de incentivo e de disponibilidade para o docente participar das atividades de atualização, a burocratização para o acesso à obtenção de apoio para a produção dos materiais, ou também, o desinteresse de alguns professores na sua atualização profissional, tanto na área tecnológica como pedagógica. Concorda-se com o fato de que o professor no Ensino Superior deve estar duplamente focado na construção do conhecimento na sua área de atuação profissional, as quais são a área da enfermagem e a qualificação permanente como docente (14).

Frente ao exposto, questionam-se quais, efetivamente, tem sido as estratégias para o desenvolvimento de tais investimentos institucionais. Para que ocorra a difusão das TED, alinhadas com a proposta pedagógica dos currículos dos cursos de Enfermagem, não basta somente a intenção dos gestores, mas sim a aplicação de recursos técnicos e humanos como equipamentos e profissionais especializados na área para suporte, além da promoção da educação permanente dos docentes.

A EAD vem sendo uma estratégia de democratização de saberes, possibilitando a formação de recursos humanos nos próprios locais de trabalho, enfim dando a dimensão da educação permanente na área da saúde como uma mudança nas maneiras de ensinar e de aprender (15). Esse movimento é percebido por docentes de Enfermagem de universidades brasileiras como sendo uma necessidade de manterem-se acompanhando as evoluções tecnológicas para assim aproximarem-se dos seus alunos (16).

Assim, mesmo sendo a modalidade EAD incipiente no ensino de enfermagem no Brasil, há relatos de franca expansão de ações em outros países os quais procuram atingir todos os segmentos do ensino com diferentes recursos tecnológicos (17-20). Nesses países são utilizados TED no ensino presencial e atividades na modalidade EAD, com diferentes orientações pedagógicas. A importância está em avaliarem-se continuamente as ações desenvolvidas, a adequação do material e a satisfação dos usuários envolvidos no processo de ensino.

Quanto a maioria dos professores terem manifestado desconhecimento em relação ao referencial pedagógico que orienta suas atividades de ensino, esse fato reforça mais uma vez a necessidade do investimento em educação permanente para professores que atuam no ensino superior, pois os mesmos são graduados em Enfermagem e a formação pedagógica nem sempre esteve presente na sua qualificação.

Os professores do presente estudo, residentes no interior do Estado, citaram a importância de serem oferecidos Cursos stricto sensu na modalidade EAD reconhecidos pela CAPES, como uma forma de facilitar a qualificação docente, reforçando a associação das TED com a educação permanente. No Brasil essa modalidade de ensino não está autorizada para oferta de Cursos stricto sensu, no entanto existem relatos no exterior de que são realizados Cursos de Mestrado em Enfermagem na modalidade a distância e estão em processo de construção das melhores práticas para orientar sua execução (19).

Apesar das inquietações com a modalidade EAD, observou-se que as TED estão presentes no cotidiano das salas de aula presenciais. Os vídeos foram os recursos mais referidos, juntamente com os ambientes virtuais de aprendizagem. Esses últimos são recursos que facilitam a publicação de materiais e a comunicação entre o professor e os alunos, em um momento histórico no qual as pessoas estão frequentemente conectadas no meio virtual, e especialmente os alunos, buscam informações por esses acessos (1).

Esse estudo teve o propósito de descrever 
a utilização de TED no ensino de Enfermagem nas Instituições de ensino superior no Estado do Rio Grande do Sul. Evidenciouse os esforços institucionais em oferecer infraestrutura tecnológica e/ou pedagógica para o atendimento da demanda crescente no uso da informática no ensino, embora muitas vezes não estejam sendo efetivas junto aos professores.

Foi possível perceber que as ações de EAD ainda são limitadas na Enfermagem, mas o uso das TED no ensino presencial é destacado, principalmente com os recursos de vídeos e de ambientes virtuais de aprendizagem, o que sinaliza a valorização da comunicação online entre os professores e os alunos.

A expansão da utilização das TED não está relacionada somente com a ampliação dos recursos institucionais, mas também de como as mesmas irão articular-se em ações interinstitucionais. As ações conjuntas destacadas pelos coordenadores de Cursos de Enfermagem podem ser uma estratégia para $\mathrm{o}$ atendimento de qualidade e de abrangência estadual.

Salienta-se como limitação do presente estudo a dificuldade de acesso às instituições que estavam relacionadas no website do MEC. Os endereços eletrônicos e telefônicos constantes nas mesmas eram imprecisos ou incompletos dificultando o contato. Cabe ainda destacar que apesar do contato realizado com as instituições, a grande maioria delas, não retornou os questionários, o que limitou a caracterização mais abrangente dos resultados. Esse dado também pode ser um indício da necessidade de estudos que permitam caracterizar os reais motivos da não participação. Esse estudo evidenciou também que o desafio ainda é grande quando se trata de conhecer como e de que forma o ensino superior na área da Enfermagem está preparado para formar um profissional realmente inserido no mundo do trabalho com competência e conhecimento.

\section{REFERÊNCIAS}

1. Wood A. Using emerging Technologies to enhance learning. Nurs Sci Q 2010 Apr; 23(2): 173-9.

2. Moule P, Ward R, Lockyer L. Nursing and healthcare students' experiences and use of e-learning in higher education. J Adv Nurs. 2010; 66(12): 2785-95.

3. Silveira DT, Catalan VM, Neutzling AL, Martinato LHM. Objetos educacionais na consulta de enfermagem: avaliação da tecnologia por estudantes de graduação. Rev Lat Am Enfermagem. 2010; 18(5): 1005-1012.

4. Ferreira PB, Cohrs CR, De Domenico EBL. Software CMAP TOOLS $^{\circledR}$ para a construção de mapas conceituais: a avaliação dos estudantes de enfermagem. Rev Esc Enferm USP 2012; 46(4): 967-72.

5. Rangel EML, Mendes IAC, Cárnio EC, Alves LMM, Crispim JA, Mazzo A, Andrade JX, Trevizan MA, Rangel AL. Avaliação, por graduandos de enfermagem, de ambiente virtual de aprendizagem para ensino de fisiologia endócrina. Acta Paul Enferm. 2011; 24(3): 327-33.

6. Fonseca LMM, Aredes NDA, Leite AM, Santos CB, Lima RAG, Scochi CGS. Avaliação de uma tecnologia educacional para a avaliação clínica de recém-nascidos prematuros. Rev Lat Am Enfermagem. 2013; 21(1): 363-370.

7. Canever BP, Prado ML, Backes VMS, Gomes DC. Produção do conhecimento acerca da formação do enfermeiro na América Latina. Rev Gaucha Enferm. 2012; 33(4): 211-220.

8. Conselho Nacional de Educação (BR). Câmara de Educação Superior. Resolução No 3, de sete de novembro de 2001. Institui diretrizes curriculares nacionais do curso de graduação em Enfermagem. Diário Oficial da União, Brasília (DF); 
2001 [citado 21 jul 2013]. Disponível em: http://portal.mec.gov.br/cne/arquivos/ pdf/CES03.pdf

9. Perrenoud P. Desenvolver competências ou ensinar saberes? A escola que prepara para a vida. Porto Alegre: Penso, 2013.

10. Rodrigues RCV, Peres HHC. Panorama brasileiro do ensino de enfermagem online. Rev Esc Enferm USP 2008; 42(2): 298-304.

11. Rojo PT, Vieira SS, Zem-Mascarenhas SH, Sandor ER, Vieira CRSP. Panorama of nursing distance education in Brazil. Rev Esc Enferm USP. 2011; 45(6): 147680.

12. Denzin NK, Lincoln YS. O planejamento da pesquisa qualitativa: teorias e abordagens. Porto Alegre; Artmed, 2006. p. 1541.

13. Brasil. Conselho Nacional de Saúde. Resolução 196 de 10 de outubro de 1996. Diretrizes e normas regulamentadoras de pesquisa em seres humanos. 9 p. 1996.

14. Pereira WR, Tavares CMM. Práticas pedagógicas no ensino de enfermagem: um estudo na perspectiva da análise institucional Rev Esc Enferm USP 2010; 44(4): 1077-84.

15. Oliveira MAN Educação à Distância como estratégia para a educação perma- nente: possibilidades e desafios. Rev Bras Enferm. 2007; 60(5): 585-9.

16. Cogo ALP, Eva Pedro ENR, da Silva APSS, Valli GP, Specht AM. Tecnologias digitais no ensino de graduação em enfermagem: as possibilidades metodológicas por docentes. Rev. Eletr. Enf. [Internet]. 2011 out/dez; [citado $21 \mathrm{dez} 2011$ ]; 13(4): 65764. Disponível em: http://www.fen.ufg. br/revista/v13/n4/v13n4a09.htm.

17. Giddens JF, Shuster G, Roehrig N. Early Student Outcomes Associated with a Virtual Community for Learning. J Nurs Educ. 2010; 49(6): 355-58.

18. Santy J, Beadle M, Needham Y. Using an online case conference to facilitate interprofessional learning. Nurse Educ Pract. 2009; 9: 383-387.

19. Avery MD; Cohen BA, Walker JD. Evaluation of an Online Graduate Nursing Curriculum: Examining Standards of Quality. Int J Nurs Educ. Scholarsh [Internet]. 2008 [cited $21 \mathrm{dez} 2011$ ]; 5(1). Disponível em: http://www.ncbi.nlm. gov/pmc/articles/PMC2920736/

20. Pfefferle PI, Den Stock EV, Nauerth A. The LEONARDO-DA-VINCI pilot project "e-learning-assistant" -Situationbased learning in nursing education. Nurse Educ Today. 2010; 30: 411-419. 